\title{
EHealth for Sedentary Behavior
}

National Cancer Institute

\section{Source}

National Cancer Institute. eHealth for Sedentary Behavior. NCI Thesaurus. Code C156708.

The use of websites, applications, or other electronic means to induce individuals to partake in spontaneous movement and reduce their sedentary behavior. 